\title{
Modified Function Projective Synchronization for a Partially Linear and Fractional-Order Financial Chaotic System with Uncertain Parameters
}

\author{
Yehong Yang and Guohua Cao \\ College of Economics \& Business Administration, Chongqing University, Chongqing 400030, China \\ Correspondence should be addressed to Guohua Cao; caoguohua@cqu.edu.cn
}

Received 1 June 2017; Revised 1 August 2017; Accepted 16 August 2017; Published 19 September 2017

Academic Editor: Jorge E. Macías-Díaz

Copyright (C) 2017 Yehong Yang and Guohua Cao. This is an open access article distributed under the Creative Commons Attribution License, which permits unrestricted use, distribution, and reproduction in any medium, provided the original work is properly cited.

\begin{abstract}
This paper investigates the modified function projective synchronization between fractional-order chaotic systems, which are partially linear financial systems with uncertain parameters. Based on the stability theory of fractional-order systems and the Lyapunov matrix equation, a controller is obtained for the synchronization between fractional-order financial chaotic systems. Using the controller, the error systems converged to zero as time tends to infinity, and the uncertain parameters were also estimated so that the phenomenon of parameter distortion was effectively avoided. Numerical simulations demonstrate the validity and feasibility of the proposed method.
\end{abstract}

\section{Introduction}

In recent years, study on chaos is one of the most interesting research topics in real and physical systems [1-4]. In 1985, chaotic behavior was discovered in financial systems [5]. In fact, chaotic phenomena often appear in financial systems [6], such as the supernormal shock of the international oil prices, stock market crash, and financial crisis. Owing to its randomness and unpredictability, these chaotic phenomena usually lead to financial crisis and instability in the financial system. Since financial risks come from uncertainties, it is important to introduce unknown parameters in financial systems $[7,8]$. Therefore, chaotic behaviors and uncertainties in financial systems must be taken into account [9]. It is necessary to investigate chaos control strategies for financial systems to address financial crisis and other related problems. Hence, it is important to achieve a synchronized and healthy development of financial markets.

Since Pecora and Carroll [10] proposed synchronization between two chaotic systems in 1990, chaotic synchronization has been extensively and intensively studied in various fields $[11,12]$. Different types of chaotic synchronization have been reported, including generalized synchronization [13], lag synchronization [14], function projective synchronization [15], modified projective synchronization [16], and modified function projective synchronization (MFPS) $[17,18]$. The modified function projective synchronization (MFPS) means that the drive and response systems could be synchronized up to a scaling function matrix, but not a constant matrix. Recent studies on complicated financial systems have shown satisfactory results using the nonlinear method [19]. Chaos synchronization in fractional-order financial systems has also been studied in recent years [20-22].

The synchronization of financial systems indicates that the financial systems in two different areas are to maintain synchronized development by the appropriate control conditions. That is, the drive system and the response system can be interpreted as a virtual economic target and a controlled objective, respectively. Hence, the goal of synchronization is to control a specific objective to a virtual economic target using the proposed controller. The dynamical behaviors of a financial system are more complex. Therefore, financial systems are not always completely synchronized, and some complex synchronization methods [20-22], such as function 
projective synchronization and MFPS, should be considered. Meanwhile, because of the complexity of the financial environment, this study highlights its theoretical value and ensures the comprehensiveness of the theory.

Also, in practice, the parameter distortion phenomenon frequently appears in fractional-order chaotic systems. In other words, parameters may be uncertain or drift with time in the chaotic synchronization between the response and drive systems because of various kinds of interferences. Many projective synchronization methods of chaotic systems with unknown parameters have been proposed in recent years [23]. Hence, achieving synchronization and identifying parameters are very important in financial chaotic systems.

In this study, we investigated fractional-order financial systems proposed by Chen [24]. A new controller is proposed to achieve synchronization. The controller makes the drive and response systems remain asymptotically stable. Based on the stability theory of fractional-order systems and the Lyapunov matrix equation, the MFPS was realized for partially linear and fractional-order (PLFO) financial chaotic systems, and the unknown parameters were also estimated so that the phenomenon of parameter distortion was avoided. Numerical simulation results showed that the proposed method effectively eliminated chaos and stabilized two financial systems.

The rest of the paper is organized as follows. In Section 2, the definition of MFPS, the PLFO chaotic system, and a useful lemma are presented. In Section 3, the scheme of MFPS is presented for PLFO chaotic systems with uncertain parameters. Numerical simulations are presented in Section 4. Finally, a conclusion is drawn in Section 5.

\section{Preliminaries}

The definitions of PLFO chaotic system and coupled PLFO chaotic system were given in [25]. We will give the definition of PLFO chaotic system with uncertain parameters and coupled PLFO chaotic system with uncertain parameters as follows.

Definition 1. The fractional-order chaotic systems with uncertain parameters are defined as follows:

$$
\begin{aligned}
& \frac{\mathrm{d}^{q} u}{\mathrm{~d} t^{q}}=M(z, \theta) \cdot u, \\
& \frac{\mathrm{d}^{q} u}{\mathrm{~d} t^{q}}=f(u, z) .
\end{aligned}
$$

It is called an uncertain PLFO chaotic system, where the state vector is $u=\left(u_{1}, u_{2}, \ldots, u_{n}\right)^{T} \in R^{n}, z \in R$ is a variable, and $f: R \times R^{n} \rightarrow R$ is a differentiable function. Its order is subject to $0<q \leq 1 . \theta \in R^{n}$ represents the vector of uncertain parameters. The coefficient matrix $M(z, \theta) \in R^{n \times n}$ is dependent on the variable $z$ and the uncertain parameter $\theta$.
System (1) can be written as

$$
\begin{aligned}
& \frac{\mathrm{d}^{q} u_{1}}{\mathrm{~d} t^{q}}=m_{11}(z, \theta) u_{1}+m_{12}(z, \theta) u_{2}+\cdots \\
& +m_{1 n}(z, \theta) u_{n} \\
& \frac{\mathrm{d}^{q} u_{2}}{\mathrm{~d} t^{q}}=m_{21}(z, \theta) u_{1}+m_{22}(z, \theta) u_{2}+\cdots \\
& +m_{2 n}(z, \theta) u_{n} \\
& \frac{\mathrm{d}^{q} u_{n}}{\mathrm{~d} t^{q}}=m_{n 1}(z, \theta) u_{1}+m_{n 2}(z, \theta) u_{2}+\cdots \\
& +m_{n n}(z, \theta) u_{n} \\
& \frac{\mathrm{d}^{q} z}{\mathrm{~d} t^{q}}=f(u, z)
\end{aligned}
$$

where $m_{i j}(z, \theta)$ is a coefficient of $u_{j}$ in the $i$ th differential equation, $i, j=1,2, \ldots, n$. Hence, $M(z, \theta)$ can be described by

$$
\begin{aligned}
& M(z, \theta) \\
& \quad=\left(\begin{array}{cccc}
m_{11}(z, \theta) & m_{12}(z, \theta) & \cdots & m_{1 n}(z, \theta) \\
m_{21}(z, \theta) & m_{22}(z, \theta) & \cdots & m_{2 n}(z, \theta) \\
\vdots & \vdots & \vdots & \vdots \\
m_{n 1}(z, \theta) & m_{n 2}(z, \theta) & \cdots & m_{n n}(z, \theta)
\end{array}\right) .
\end{aligned}
$$

Obviously, system (1) is an uncertain partially linear and integer-order chaotic system with $q=1$.

Definition 2. Consider two uncertain PLFO chaotic systems (1), which can be described as

$$
\begin{aligned}
\frac{\mathrm{d}^{q} u_{r}}{\mathrm{~d} t^{q}} & =M(z, \theta) \cdot u_{r}, \\
\frac{\mathrm{d}^{q} z}{\mathrm{~d} t^{q}} & =f\left(u_{r}, z\right), \\
\frac{\mathrm{d}^{q} u_{s}}{\mathrm{~d} t^{q}} & =\widetilde{M}(z, \tilde{\theta}) \cdot u_{s}+\psi .
\end{aligned}
$$

The system is coupled by the variable $z$. The subscripts $r$ and $s$ in system (4) represent the drive and response systems, respectively. $\psi \in R^{n}$ is a controller. $\tilde{\theta}$ is the estimated value of unknown parameters. The coefficient matrix $\widetilde{M}(z, \widetilde{\theta}) \in R^{n \times n}$ is dependent on $z$ and $\tilde{\theta}$. System (4) is the coupled PLFO chaotic system with uncertain parameters [26]. 
The synchronization error vector of the MFPS is defined as

$$
e(t)=u_{s}-h(t) \cdot u_{r}
$$

where $h(t)=\operatorname{diag}\left\{h_{1}(t), h_{2}(t), \ldots, h_{n}(t)\right\}, h_{i}(t)$ are continuously differentiable functions, and $h(t)$ is a scaling function matrix. $e(t)=\left(e_{1}(t), e_{2}(t), \ldots, e_{n}(t)\right)^{T} \in R^{n}$ is an error state vector.

Definition 3. System (4) is said to be MFPS with respect to the scaling function matrix $h(t)$ if there exists a vector controller $\psi$ such that

$$
\lim _{t \rightarrow \infty}\|e(t)\|=\lim _{t \rightarrow \infty}\left\|u_{s}-h(t) \cdot u_{r}\right\|=0
$$

where $\|\cdot\|$ is the Euclidean norm. It implies that the MFPS between the drive and response system (4) can be achieved or the error dynamic systems are globally asymptotically stable.

Remark 4. If $h_{1}(t)=h_{2}(t)=\cdots=h_{n}(t)$, the MFPS converts to function projective synchronization. If $h_{1}(t), h_{2}(t), \ldots, h_{n}(t)$ represent identical or different constants, the MFPS is simplified to modified projective synchronization (such as antisynchronization and complete synchronization). If $h_{1}(t)=h_{2}(t)=\cdots=h_{n}(t)=0$, the synchronization problem is transformed into a control problem.

Lemma 5 (see [27]). Presume that the fractional-order autonomous system is

$$
\frac{\mathrm{d}^{q} e}{\mathrm{~d} t^{q}}=A(e) \cdot e, \quad e(0)=e_{0},
$$

where $q_{i} \in(0,1](i=1,2, \ldots, n)$ are the orders of the fractional derivative and $q=\left(q_{1}, q_{2}, \ldots, q_{n}\right)^{T}, A(e) \in R^{n \times n}$ is a coefficient matrix which is a polynomial matrix and depends on the state vector e, and $e=\left(e_{1}, e_{2}, \ldots, e_{n}\right)^{T} \in R^{n}$ is an $n$-dimension state vector. System (7) is asymptotically stable if and only if

$$
|\arg (\lambda)| \geq \frac{\pi \alpha}{2}, \quad \alpha=\max \left(q_{1}, q_{2}, \ldots, q_{n}\right),
$$

where $\lambda$ is an arbitrary eigenvalue of $A(e)$. In this case, each state vector decays toward 0 , such as $t^{-\alpha}$. Furthermore, the system is stable if and only if $|\arg (\lambda)| \geq \pi \alpha / 2$ and the critical eigenvalues that satisfy $|\arg (\lambda)|=\pi \alpha / 2$ have geometric multiplicity one.

Then, the MFPS between the drive and response system (4) is transformed into the analysis of the asymptotical stability of zero solution of the error system (7).
Theorem 6 (see [28]). Given the autonomous system (7), there exist a real symmetric positive definite matrix $P$ and a positive definite matrix $Q$ such that the MFPS between the drive and response system (4) can be achieved if $P A(e)+(A(e))^{\mathrm{H}} P=-Q$, where $\mathrm{H}$ is the conjugate transpose of a matrix and $P A(e)+$ $(A(e))^{\mathrm{H}} P=-Q$ is called a continuous Lyapunov matrix equation.

Proof. Assume that $\lambda$ is one of the eigenvalues of the polynomial matrix $A(e)$ and the corresponding nonzero eigenvector is $\beta$; that is,

$$
A(e) \beta=\lambda \beta \text {. }
$$

For (9), the Hermitian transpose is

$$
\overline{(A(e) \beta)^{T}}=\bar{\lambda} \beta^{\mathrm{H}} .
$$

Multiplying the left side of (9) by $\beta^{\mathrm{H}} P$, we can obtain

$$
\beta^{\mathrm{H}} P A(e) \beta=\lambda \beta^{\mathrm{H}} P \beta .
$$

Then, multiplying the right side of (10) by $P \beta$, we can also obtain

$$
\beta^{\mathrm{H}}(A(e))^{\mathrm{H}} P \beta=\bar{\lambda} \beta^{\mathrm{H}} P \beta .
$$

From (11) and (12), we obtain

$$
\beta^{\mathrm{H}}\left(P A(e)+(A(e))^{\mathrm{H}} P\right) \beta=(\lambda+\bar{\lambda}) \beta^{\mathrm{H}} P \beta .
$$

Since $\left(P A(e)+(A(e))^{\mathrm{H}} P\right)^{\mathrm{H}}=P A(e)+(A(e))^{\mathrm{H}} P$, then $P A(e)+$ $(A(e))^{\mathrm{H}} P$ is a Hermitian matrix. At the same time, since $P A(e)+(A(e))^{\mathrm{H}} P=-Q, P$ is a real symmetric positive definite matrix, and $Q$ is a positive definite matrix, then $\beta^{\mathrm{H}} P \beta>0$ and $\beta^{\mathrm{H}}(-\mathrm{Q}) \beta<0$, so that

$$
\lambda+\bar{\lambda}=\frac{\beta^{\mathrm{H}}(-\mathrm{Q}) \beta}{\beta^{\mathrm{H}} P \beta}<0 .
$$

From (14), we can obtain $|\arg (\lambda)|>\pi / 2 \geq \pi \alpha / 2(\alpha \leq 1)$. According to Lemma 5, the equilibrium point of system ( 7 ) is asymptotically stable. That is,

$$
\lim _{t \rightarrow \infty}\|e(t)\|=\lim _{t \rightarrow \infty}\left\|u_{s}-h(t) \cdot u_{r}\right\|=0
$$

which indicates that the MFPS between the drive and response system (4) can be achieved. The proof is completed. 
Remark 7. Since $A(e)$ is a polynomial matrix, it is difficult to solve for the eigenvalues. However, based on Theorem 6, the equilibrium point in system (7) is asymptotically stable if and only if $P A(e)+(A(e))^{\mathrm{H}} P=-Q$ is a continuous Lyapunov matrix equation.

\section{Designing the Lyapunov Matrix Equation}

Next, we introduce a scheme of MFPS for coupled PLFO chaotic systems with uncertain parameters (7).

Theorem 8. For a given scaling function matrix $h(t)$, we can select a controller (16) and a synchronization error system (17) as follows:

$$
\begin{aligned}
\psi= & -h(t) \widetilde{M}(z, \widetilde{\theta}) u_{r}-M(z, \theta) e+K e \\
& +\frac{\mathrm{d}^{q}\left(h(t) u_{r}\right)}{\mathrm{d} t^{q}}, \\
\frac{\mathrm{d}^{q} e}{\mathrm{~d} t^{q}}= & {[\widetilde{M}(z, \widetilde{\theta})-M(z, \theta)+K] e, }
\end{aligned}
$$

where $K=\operatorname{diag}\left\{k_{1}, k_{2}, \ldots, k_{n}\right\}, k_{i}<0,0<q \leq 1$. The error system (17) can be equivalently written as

$$
\begin{aligned}
& \frac{\mathrm{d}^{q} e_{1}}{\mathrm{~d} t^{q}}=A_{11} e_{\theta_{11}}+A_{12} e_{\theta_{12}}+\cdots+A_{1 l_{1}} e_{\theta_{1 l_{1}}}+k_{1} e_{1}, \\
& \frac{\mathrm{d}^{q} e_{2}}{\mathrm{~d} t^{q}}=A_{2\left(l_{1}+1\right)} e_{\theta_{2\left(l_{1}+1\right)}}+A_{2\left(l_{1}+2\right)} e_{\theta_{2\left(l_{1}+2\right)}}+\cdots \\
& +A_{2 l_{2}} e_{\theta_{2 l_{2}}}+k_{2} e_{2}, \\
& \vdots \\
& \frac{\mathrm{d}^{q} e_{n}}{\mathrm{~d} t^{q}}=A_{n\left(l_{n-1}+1\right)} e_{\theta_{n\left(l_{n-1}+1\right)}}+A_{n\left(l_{n-1}+2\right)} e_{\theta_{n\left(l_{n-1}+2\right)}}+\cdots \\
& \quad+A_{n l_{n}} e_{\theta_{n l_{n}}}+k_{n} e_{n},
\end{aligned}
$$

where $e_{\theta_{i j}}=\widetilde{\theta}_{i j}-\theta_{i j}\left(i=1,2, \ldots, n, j=1,2, \ldots, l_{1}, l_{1}+\right.$ $1, \ldots, l_{n-1}, l_{n-1}+1, \ldots, l_{n}$, where $l_{1}$ stands for the number of unknown parameters in the first error equation, and $l_{n}-l_{n-1}$ stands for the number of unknown parameters in the nth error equation) and $A_{i j}$ is a coefficient of $e_{\theta_{i j}}$ in the ith error equation. Therefore, we can obtain the systems with uncertain parameters as

$$
\begin{aligned}
& \frac{\mathrm{d}^{q} e_{\theta_{11}}}{\mathrm{~d} t^{q}}=-A_{11} e_{1}+k_{1}^{\prime} e_{\theta_{11}}, \\
& \frac{\mathrm{d}^{q} e_{\theta_{12}}}{\mathrm{~d} t^{q}}=-A_{12} e_{1}+k_{2}^{\prime} e_{\theta_{12}},
\end{aligned}
$$

$$
\begin{gathered}
\frac{\mathrm{d}^{q} e_{\theta_{1 l_{1}}}}{\mathrm{~d} t^{q}}=-A_{1 l_{1}} e_{1}+k_{l_{1}}^{\prime} e_{\theta_{1 l_{1}}}, \\
\frac{\mathrm{d}^{q} e_{\theta_{2\left(l_{1}+1\right)}}}{\mathrm{d} t^{q}}=-A_{2\left(l_{1}+1\right)} e_{2}+k_{\left(l_{1}+1\right)}^{\prime} e_{\theta_{2\left(l_{1}+1\right)}},
\end{gathered}
$$

$$
\begin{gathered}
\frac{\mathrm{d}^{q} e_{\theta_{2 l_{2}}}}{\mathrm{~d} t^{q}}=-A_{2 l_{2}} e_{2}+k_{l_{2}}^{\prime} e_{\theta_{2 l_{2}}}, \\
\vdots \\
\frac{\mathrm{d}^{q} e_{\theta_{n l_{n}}}}{\mathrm{~d} t^{q}}=-A_{n l_{n}} e_{n}+k_{l_{n}}^{\prime} e_{\theta_{n l_{n}}},
\end{gathered}
$$

where $k_{j}^{\prime}<0\left(j=1,2, \ldots, l_{n}\right)$. We can achieve the MFPS of system (7) through the error system of (18) and the estimate of the system with unknown parameters (19). That is, the error system and the estimate of unknown parameters are asymptotically stable.

Proof. Assume that $e(t)=u_{s}-h(t) \cdot u_{r}$; we have

$$
\begin{aligned}
\frac{\mathrm{d}^{q} e(t)}{\mathrm{d} t^{q}} & =\frac{\mathrm{d}^{q} u_{s}}{\mathrm{~d} t^{q}}-\frac{\mathrm{d}^{q}\left(h(t) u_{r}\right)}{\mathrm{d} t^{q}} \\
& =\widetilde{M}(z, \widetilde{\theta}) u_{s}+\psi-\frac{\mathrm{d}^{q}\left(h(t) u_{r}\right)}{\mathrm{d} t^{q}} .
\end{aligned}
$$

We select $\psi=-h(t) \widetilde{M}(z, \widetilde{\theta}) u_{r}-M(z, \theta) e+K e+\mathrm{d}^{q}\left(h(t) u_{r}\right) / \mathrm{d} t^{q}$; then it has

$$
\begin{aligned}
\frac{\mathrm{d}^{q} e(t)}{\mathrm{d} t^{q}}= & \widetilde{M}(z, \widetilde{\theta}) u_{s}+\psi-\frac{\mathrm{d}^{q}\left(h(t) u_{r}\right)}{\mathrm{d} t^{q}} \\
= & \widetilde{M}(z, \widetilde{\theta}) u_{s}-h(t) \widetilde{M}(z, \widetilde{\theta}) u_{r}-M(z, \theta) e \\
& +K e+\frac{\mathrm{d}^{q}\left(h(t) u_{r}\right)}{\mathrm{d} t^{q}}-\frac{\mathrm{d}^{q}\left(h(t) u_{r}\right)}{\mathrm{d} t^{q}} \\
= & {[\widetilde{M}(z, \widetilde{\theta})-M(z, \theta)+K] e . }
\end{aligned}
$$

Hence, we obtain a controller (16) and a synchronization error system (17).

From (18) and (19), the error system and the estimate of unknown parameters can be simply written as

$$
\left(\begin{array}{c}
\frac{\mathrm{d}^{q} e}{\mathrm{~d} t^{q}} \\
\frac{\mathrm{d}^{q} e_{\theta}}{\mathrm{d} t^{q}}
\end{array}\right)=A\left(\begin{array}{c}
e \\
e_{\theta}
\end{array}\right),
$$

where $e=\left(e_{1}, e_{2}, \ldots, e_{n}\right)^{T}, e_{\theta}=\left(e_{\theta_{11}}, e_{\theta_{12}}, \ldots, e_{\theta_{1 l_{1}}}, e_{\theta_{2\left(l_{1}+1\right)}}\right.$, $\left.\ldots, e_{\theta_{2 l_{2}}}, \ldots, e_{\theta_{n l_{n}}}\right)^{T}$, and 


$$
A=\left(\begin{array}{cccccccccccccc}
k_{1} & 0 & \cdots & 0 & A_{11} & \cdots & A_{1 l_{1}} & 0 & \cdots & 0 & \cdots & 0 & \cdots & 0 \\
0 & k_{2} & \cdots & 0 & 0 & \cdots & 0 & A_{2\left(l_{1}+1\right)} & \cdots & A_{2 l_{2}} & \cdots & 0 & \cdots & 0 \\
\vdots & \vdots & \ddots & \vdots & \vdots & \vdots & \vdots & \vdots & \vdots & \vdots & \cdots & \vdots & \vdots & \vdots \\
0 & 0 & \cdots & k_{n} & 0 & \cdots & 0 & 0 & \cdots & 0 & \vdots & A_{n\left(l_{n-1}+1\right)} & \cdots & A_{n l_{n}} \\
-A_{11} & 0 & \cdots & 0 & k_{1}^{\prime} & \cdots & 0 & 0 & \cdots & 0 & \cdots & 0 & \vdots & 0 \\
\vdots & \vdots & \vdots & \vdots & \vdots & \ddots & \vdots & \vdots & \vdots & \vdots & \cdots & \vdots & \cdots & \vdots \\
-A_{1 l_{1}} & 0 & \cdots & 0 & 0 & \cdots & k_{l_{1}}^{\prime} & 0 & \cdots & 0 & \cdots & 0 & \cdots & 0 \\
0 & -A_{2\left(l_{1}+1\right)} & \cdots & 0 & 0 & \cdots & 0 & k_{l_{1}+1}^{\prime} & \cdots & 0 & \cdots & 0 & \cdots & 0 \\
\vdots & \vdots & \vdots & \vdots & \vdots & \vdots & \vdots & \vdots & \ddots & \vdots & \vdots & \vdots & \vdots & \vdots \\
0 & -A_{2 l_{2}} & \cdots & 0 & 0 & \cdots & 0 & 0 & \cdots & k_{l_{2}}^{\prime} & \cdots & 0 & \cdots & 0 \\
\vdots & \vdots & \cdots & \vdots & \vdots & \cdots & \vdots & \vdots & \cdots & \vdots & \ddots & \vdots & \cdots & \vdots \\
0 & 0 & \vdots & -A_{n\left(l_{n-1}+1\right)} & 0 & \vdots & 0 & 0 & \cdots & 0 & \cdots & k_{l_{n-1}+1}^{\prime} & \cdots & 0 \\
\vdots & \vdots & \cdots & -A_{n l_{n}} & 0 & \cdots & 0 & 0 & \cdots & 0 & \cdots & 0 & \cdots & k_{l_{n}}^{\prime}
\end{array}\right) .
$$

Hence, we can obtain $P A+A^{\mathrm{H}} P=-Q$, where $P$ is a real symmetric positive definite matrix and $Q$ is a positive definite matrix. We usually choose $P=E$, where $E$ is an identity matrix. Then,

$$
Q=\operatorname{diag}\left\{-2 k_{1},-2 k_{2}, \ldots,-2 k_{n},-2 k_{1}^{\prime}, \ldots,-2 k_{l_{n}}^{\prime}\right\} .
$$

According to Theorem $6, P A+A^{\mathrm{H}} P=-\mathrm{Q}$ is a continuous Lyapunov matrix equation. Meanwhile, we can realize the MFPS of system (7) through controller (16). Therefore, $\lim _{t \rightarrow \infty}\|e(t)\|=0$, which indicates that the MFPS within system (17) can be achieved. The proof is completed.

Remark 9. In the proof of Theorem 8 , it is easy to see that the scaling function matrix $h(t)$ has no direct effect on $\mathrm{d}^{q} e / \mathrm{d} t^{q}$ and $\mathrm{d}^{q} e_{\theta} / \mathrm{d} t^{q}$. The results verify the validity and feasibility of the proposed control strategy.

Remark 10. Based on Theorem 8, we can choose the scaling function matrix to realize a variety of synchronizations by the method. For example, if $h_{1}(t)=h_{2}(t)=\cdots=$ $h_{n}(t)$ are continuously differentiable functions, the MFPS converts to function projective synchronization, and if $h(t)=$ $\operatorname{diag}\left\{h_{1}(t), h_{2}(t), \ldots, h_{n}(t)\right\}, h_{i}(t)(i=1,2, \ldots, n)$ being real constants, the MFPS converts to modified projective synchronization (such as complete synchronization and antisynchronization).

Remark 11. This method has universal applicability if and only if the chaotic system is a PLFO chaotic system (1).

\section{Numerical Simulations}

Huang and Li [29] proposed a dynamic model of finance comprising three first-order differential equations. Then, following the dynamic model, Chen [24] proposed a fractionalorder financial system to describe the running of a financial system as follows:

$$
\begin{aligned}
\frac{\mathrm{d}^{q_{1}} x_{1}}{\mathrm{~d} t^{q_{1}}} & =n x_{3}+m x_{1} x_{2}-a x_{1}, \\
\frac{\mathrm{d}^{q_{2}} x_{2}}{\mathrm{~d} t^{q_{2}}} & =1-b x_{2}-x_{1}^{2}, \\
\frac{\mathrm{d}^{q_{3}} x_{3}}{\mathrm{~d} t^{q_{3}}} & =p x_{1}-c x_{3} .
\end{aligned}
$$

It has three nonlinear equations of fractional-order chaotic systems. The state variables $x_{1}, x_{2}, x_{3}$ represent the interest rate, the investment demand, and the price index, respectively. Changes in $x_{1}$ are mostly influenced by two factors: contradictions from the investment market, which indicate a surplus between investment and savings, and structural adjustment from prices of goods. The changing rate of $x_{2}$ is proportional to the rate of investment but inversely proportional to the cost of investment and interest rates. Changes in $x_{3}$ are controlled by a contradiction between supply and demand in commercial markets and are affected by inflation rates. Particularly, the negative interest rate $x_{1}$, negative investment demand $x_{2}$, and negative price index $x_{3}$ mean that the banks' deposit rates are below the level 


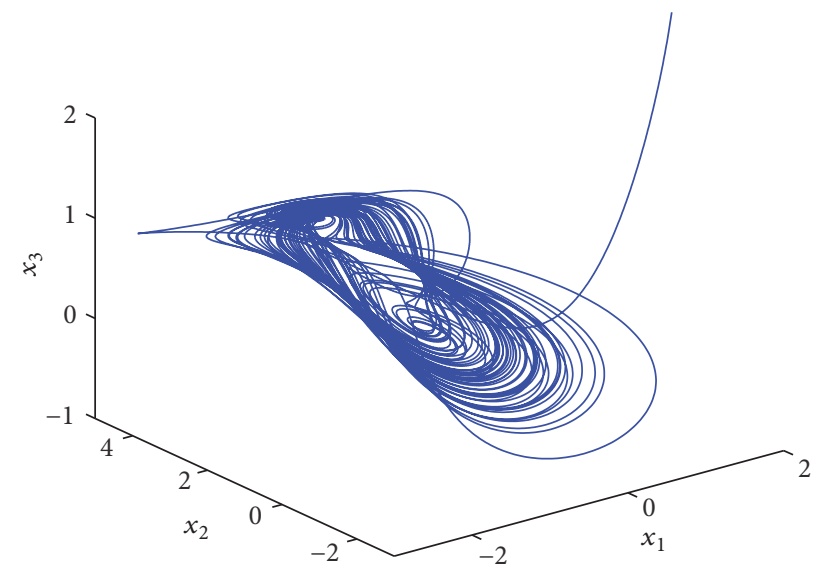

FIGURE 1: Three-dimensional phase diagram of fractional-order financial system (25).

of inflation, the investment market presents overinvestment, and the rate of deflation is increasing, respectively.

The constants $a, b, c$ are representatives of the saving amount, the per-investment cost, and the elasticity of demand of the commercial markets. The fractional-order financial system (25) will exhibit chaotic behavior when $q_{i}=q \geq$ $0.85, i=1,2,3$, and $n=1, m=1, p=-1, a=3$, $b=0.1$, and $c=1$. When $q=0.9$, the three-dimensional phase diagram of system (25) is illustrated in Figure 1 and the largest Lyapunov exponent is $L=0.14$. The results highlight the chaotic attractor of fractional-order financial system (25).

It is obvious that system (25) is a PLFO chaotic system. Therefore, the drive and response systems can be constructed as follows:

$$
\begin{aligned}
\frac{\mathrm{d}^{q_{1}} x_{1}}{\mathrm{~d} t^{q_{1}}} & =n x_{3}+m x_{1} x_{2}-a x_{1}, \\
\frac{\mathrm{d}^{q_{3}} x_{3}}{\mathrm{~d} t^{q_{3}}} & =p x_{1}-c x_{3}, \\
\frac{\mathrm{d}^{q_{2}} x_{2}}{\mathrm{~d} t^{q_{2}}} & =1-b x_{2}-x_{1}^{2}, \\
\frac{\mathrm{d}^{q_{1}} x_{4}}{\mathrm{~d} t^{q_{1}}} & =\widetilde{n} x_{5}+\widetilde{m} x_{4} x_{2}-\tilde{a} x_{4}+\psi_{1}, \\
\frac{\mathrm{d}^{q_{3}} x_{5}}{\mathrm{~d} t^{q_{3}}} & =\widetilde{p} x_{4}-\widetilde{c} x_{5}+\psi_{2},
\end{aligned}
$$

where $\psi_{i}(i=1,2)$ is the controller. In the coupled system (26), the drive system evolves independently, while the response system is governed by the drive system through the variable $x_{2}$. That is, the drive system is

$$
\begin{aligned}
\frac{\mathrm{d}^{q_{1}} x_{1}}{\mathrm{~d} t^{q_{1}}} & =n x_{3}+m x_{1} x_{2}-a x_{1}, \\
\frac{\mathrm{d}^{q_{3}} x_{3}}{\mathrm{~d} t^{q_{3}}} & =p x_{1}-c x_{3}, \\
\frac{\mathrm{d}^{q_{2}} x_{2}}{\mathrm{~d} t^{q_{2}}} & =1-b x_{2}-x_{1}^{2},
\end{aligned}
$$

and the response system is

$$
\begin{aligned}
& \frac{\mathrm{d}^{q_{1}} x_{4}}{\mathrm{~d} t^{q_{1}}}=\tilde{n} x_{5}+\widetilde{m} x_{4} x_{2}-\widetilde{a} x_{4}+\psi_{1}, \\
& \frac{\mathrm{d}^{q_{3}} x_{5}}{\mathrm{~d} t^{q_{3}}}=\tilde{p} x_{4}-\widetilde{c} x_{5}+\psi_{2} .
\end{aligned}
$$

System (26) becomes a coupled PLFO chaotic system with uncertain parameters. Based on Theorem 8 and $q_{i}(i=$ $1,2,3)=q$, we have $u_{r}=\left(\begin{array}{l}x_{1} \\ x_{3}\end{array}\right), M\left(x_{2}, \theta\right)=\left(\begin{array}{cc}m x_{2}-a & n \\ p & -c\end{array}\right)$, $\widetilde{M}\left(x_{2}, \widetilde{\theta}\right)=\left(\begin{array}{cc}\widetilde{m} x_{2}-\widetilde{a} & \widetilde{n} \\ \widetilde{p} & -\widetilde{c}\end{array}\right), h(t)=\left(\begin{array}{cc}h_{1}(t) & 0 \\ 0 & h_{2}(t)\end{array}\right), K=\left(\begin{array}{cc}k_{1} & 0 \\ 0 & k_{2}\end{array}\right)$, $e=\left(\begin{array}{l}e_{1} \\ e_{2}\end{array}\right)=\left(\begin{array}{l}x_{1}-h_{1}(t) x_{4} \\ x_{3}-h_{2}(t) x_{5}\end{array}\right), e_{m}=\widetilde{m}-m, e_{a}=\widetilde{a}-a, e_{n}=\widetilde{n}-n$, $e_{p}=\widetilde{p}-p$, and $e_{c}=\widetilde{c}-c$. According to (16), we can describe the controller as

$$
\begin{aligned}
\psi= & \left(\begin{array}{l}
\psi_{1} \\
\psi_{2}
\end{array}\right) \\
= & -h(t) \widetilde{M}\left(x_{2}, \widetilde{\theta}\right) u_{r}-M\left(x_{2}, \theta\right) e+K e \\
& +\frac{\mathrm{d}^{q}\left(h(t) u_{r}\right)}{\mathrm{d} t^{q}} .
\end{aligned}
$$

From (17) to (19), we can obtain the error systems as

$$
\begin{aligned}
& \frac{\mathrm{d}^{q} e_{1}}{\mathrm{~d} t^{q}}=x_{2} e_{1} e_{m}+\left(-e_{1}\right) e_{a}+e_{2} e_{n}+k_{1} e_{1}, \\
& \frac{\mathrm{d}^{q} e_{2}}{\mathrm{~d} t^{q}}=e_{1} e_{p}+\left(-e_{2}\right) e_{c}+k_{2} e_{2} .
\end{aligned}
$$

It shows that $A_{11}=x_{2} e_{1}, A_{12}=-e_{1}, A_{13}=e_{2}, A_{24}=e_{1}$, and $A_{25}=-e_{2}$. Therefore, the unknown parameters systems are given by

$$
\begin{aligned}
& \frac{\mathrm{d}^{q} e_{m}}{\mathrm{~d} t^{q}}=-x_{2} e_{1}^{2}+k_{1}^{\prime} e_{m}, \\
& \frac{\mathrm{d}^{q} e_{a}}{\mathrm{~d} t^{q}}=e_{1}^{2}+k_{2}^{\prime} e_{a}, \\
& \frac{\mathrm{d}^{q} e_{n}}{\mathrm{~d} t^{q}}=-e_{1} e_{2}+k_{3}^{\prime} e_{n}, \\
& \frac{\mathrm{d}^{q} e_{p}}{\mathrm{~d} t^{q}}=-e_{1} e_{2}+k_{4}^{\prime} e_{p}, \\
& \frac{\mathrm{d}^{q} e_{c}}{\mathrm{~d} t^{q}}=e_{2}^{2}+k_{5}^{\prime} e_{c}
\end{aligned}
$$

where $K^{\prime}=\operatorname{diag}\left(k_{1}^{\prime}, k_{2}^{\prime}, k_{3}^{\prime}, k_{4}^{\prime}, k_{5}^{\prime}\right)\left(k_{i}^{\prime}<0\right)$.

The initial conditions are $x_{1}(0)=-0.5, x_{2}(0)=-0.2$, $x_{3}(0)=8, x_{4}(0)=3, x_{5}(0)=-1$, and $q=0.9$ and the estimated parameters have initial conditions $\widetilde{n}(0)=-1$, $\widetilde{m}(0)=3, \widetilde{a}(0)=-0.5, \widetilde{p}(0)=3, \widetilde{c}(0)=3.5$, and $k_{i}=$ $-10(i=1,2)$ and $k_{i}^{\prime}=-20(i=1,2,3,4,5)$. Let the scaling function factors $h_{1}(t)=0.1 \cos (2 t)$ and $h_{2}(t)=\sqrt[3]{t}$; then the simulation results are shown in Figures 2 and 3. Obviously, in Figure 2, the errors converge to zero as time goes to infinity, which implies that the MFPS of system (26) is achieved with 


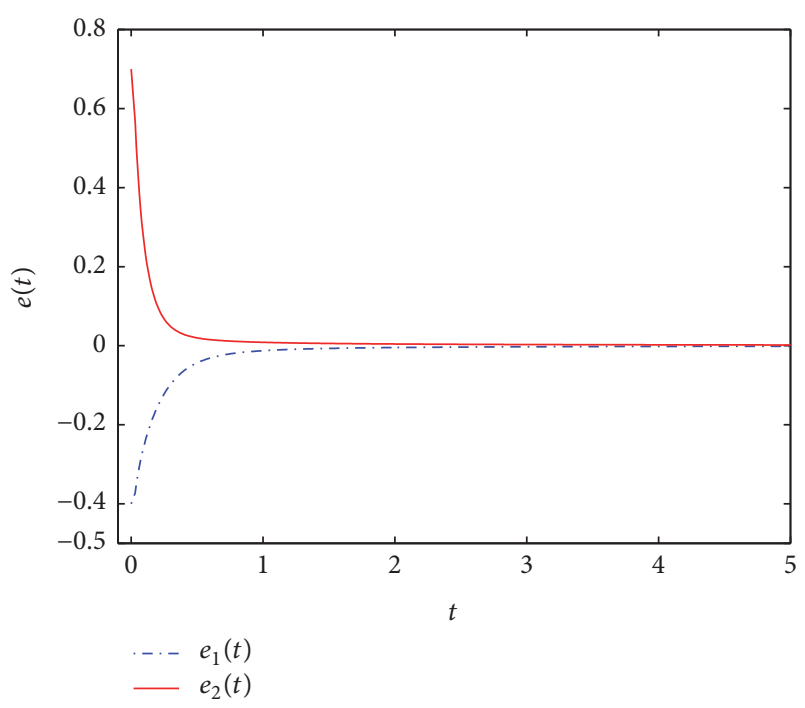

FIGURE 2: MFPS errors of system (30) with $h_{1}(t)=0.1 \cos (2 t)$ and $h_{2}(t)=\sqrt[3]{t}$.

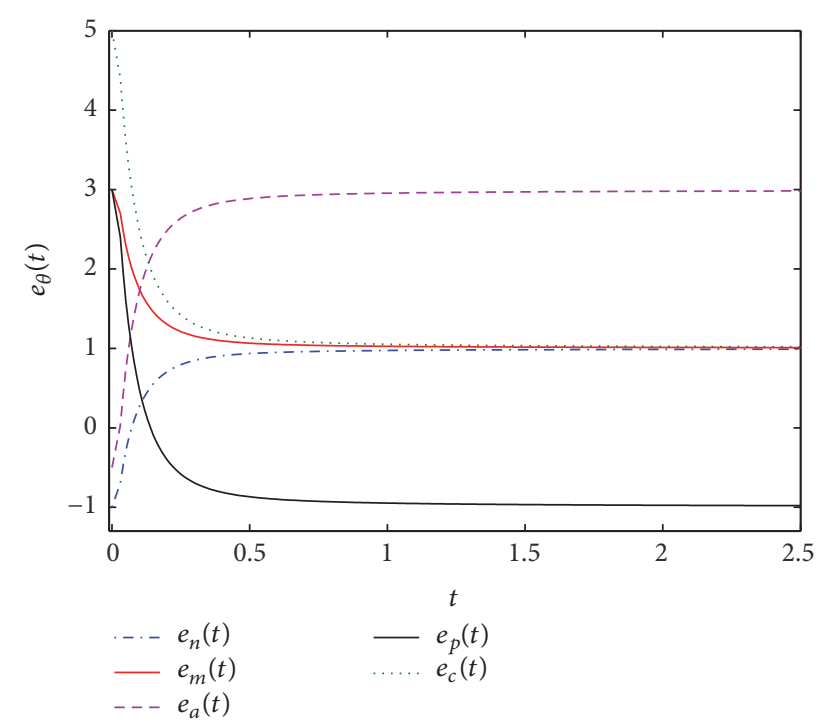

Figure 3: Unknown parameters of system (31) with $h_{1}(t)=$ $0.1 \cos (2 t)$ and $h_{2}(t)=\sqrt[3]{t}$.

the scaling function factors. Figure 3 shows that the estimated values of unknown parameters $\widetilde{n}, \widetilde{m}, \widetilde{a}, \widetilde{p}$, and $\widetilde{c}$ converge to $n=1, m=1, a=3, p=-1$, and $c=1$, respectively, as $t \rightarrow \infty$. Therefore, the uncertain parameters $n, m, a, p$, and $c$ are identified. Additionally, with the scaling function factors $h_{1}(t)=0.1 \cos (2 t)$ and $h_{2}(t)=\sqrt[3]{t}$, phase portrait of the drive and response systems is illustrated in Figure 4.

\section{Conclusion}

In this paper, the MFPS was investigated for the coupled uncertain fractional-order financial system. Based on the stability criterion of the fractional-order system and the continuous Lyapunov matrix equation, a synchronization

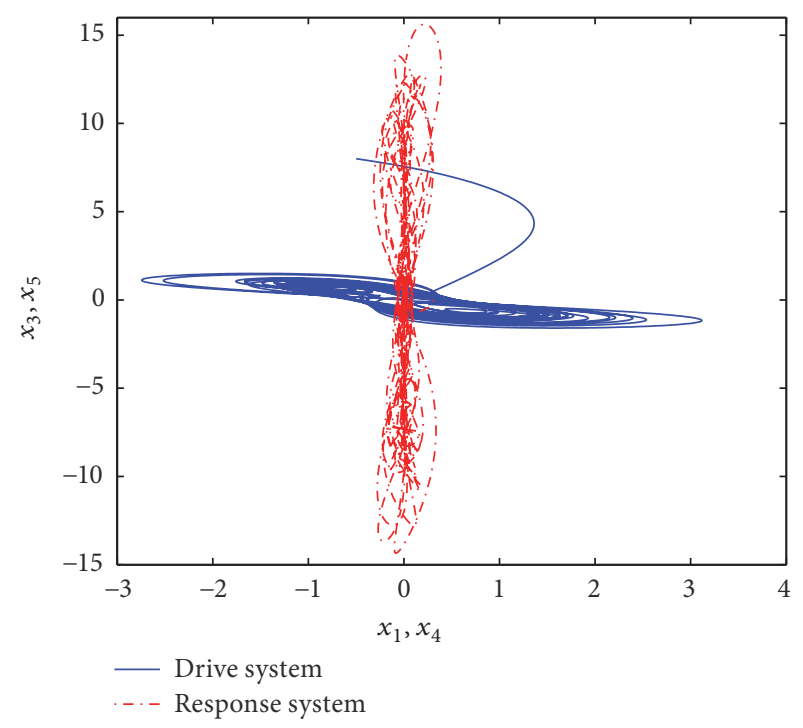

FIgURE 4: Phase portrait of the drive and response systems in $x_{1}-$ $x_{3}\left(x_{4}-x_{5}\right)$ plane with $h_{1}(t)=0.1 \cos (2 t)$ and $h_{2}(t)=\sqrt[3]{t}$.

controller was proposed. Then, using an uncertain financial system, we verified the validity of the proposed controller. The unknown parameters were also estimated so that the phenomenon of parameter distortion was effectively avoided. The results showed that the controller was feasible and effective. In theory, the proposed approach can realize a synchronized and healthy development of financial markets. In the case of possessing homogeneous investment demand, we can keep profits with price index synchronous development so that interference factors can be avoided.

\section{Conflicts of Interest}

The authors declare that they have no conflicts of interest.

\section{Acknowledgments}

This research was supported by the State Key Program of the National Natural Science of China (no. 71232004) and Fundamental Research Funds for the Central Universities of China (no. CDJSK1001).

\section{References}

[1] A. L. Chian, F. A. Zorotto, E. L. Rempel, and C. Rogers, "Attractor merging crisis in chaotic business cycles," Chaos Solitons \& Fractals, vol. 24, no. 3, pp. 869-875, 2005.

[2] A. C.-L. Chian, E. L. Rempel, and C. Rogers, "Complex economic dynamics: Chaotic saddle, crisis and intermittency," Chaos, Solitons and Fractals, vol. 29, no. 5, pp. 1194-1218, 2006.

[3] L. De Cesare and M. Sportelli, "A dynamic IS-LM model with delayed taxation revenues," Chaos, Solitons \& Fractals, vol. 25, no. 1, pp. 233-244, 2005.

[4] L. Fanti and P. Manfredi, "Chaotic business cycles and fiscal policy: an IS-LM model with distributed tax collection lags," Chaos, Solitons \& Fractals, vol. 32, no. 2, pp. 736-744, 2007. 
[5] J.-M. Grandmont, "On endogenous competitive business cycles," Econometrica, vol. 53, no. 5, pp. 995-1045, 1985.

[6] R. H. Day, Complex economic dynamics, MIT Press, Boston, 1994.

[7] C. Ma and X. Wang, "Hopf bifurcation and topological horseshoe of a novel finance chaotic system," Communications in Nonlinear Science and Numerical Simulation, vol. 17, no. 2, pp. 721-730, 2012.

[8] F. H. Knight, "Risk, uncertainty and profit," Social Science Electronic Publishing, vol. 4, pp. 682-690, 2005.

[9] Z. Wang, X. Huang, and H. Shen, "Control of an uncertain fractional order economic system via adaptive sliding mode," Neurocomputing, vol. 83, pp. 83-88, 2012.

[10] L. M. Pecora and T. L. Carroll, "Synchronization in chaotic systems," Physical Review Letters, vol. 64, no. 8, pp. 821-824, 1990.

[11] Y. Chen and X. Li, "Function projective synchronization between two identical chaotic systems," International Journal of Modern Physics C, vol. 18, no. 5, pp. 883-888, 2007.

[12] Y. Chen, H. An, and Z. Li, "The function cascade synchronization approach with uncertain parameters or not for hyperchaotic systems," Applied Mathematics and Computation, vol. 197, no. 1, pp. 96-110, 2008.

[13] S. S. Yang and C. K. Duan, "Generalized synchronization in chaotic systems," Chaos, Solitons and Fractals, vol. 9, no. 10, pp. 1703-1707, 1998.

[14] L. Wang, Z. Yuan, X. Chen, and Z. Zhou, "Lag synchronization of chaotic systems with parameter mismatches," Communications in Nonlinear Science and Numerical Simulation, vol. 16, no. 2, pp. 987-992, 2011.

[15] H. Du, Q. Zeng, and C. Wang, "Function projective synchronization of different chaotic systems with uncertain parameters," Physics Letters, Section A: General, Atomic and Solid State Physics, vol. 372, no. 33, pp. 5402-5410, 2008.

[16] S.-J. Ma, Q. Shen, and J. Hou, "Modified projective synchronization of stochastic fractional order chaotic systems with uncertain parameters," Nonlinear Dynamics, vol. 73, no. 1-2, pp. 93-100, 2013.

[17] Z. G. Li and W. Xu, "Adaptive modified projective synchronization and parameter estimation for chaotic systems with uncertain parameters," Gongcheng Shuxue Xuebao. Chinese Journal of Engineering Mathematics, vol. 27, no. 1, pp. 30-36, 2010.

[18] H. Du and P. Shi, "A new robust adaptive control method for modified function projective synchronization with unknown bounded parametric uncertainties and external disturbances," Nonlinear Dynamics. An International Journal of Nonlinear Dynamics and Chaos in Engineering Systems, vol. 85, no. 1, pp. 355-363, 2016.

[19] I. Pan, S. Das, and S. Das, "Multi-objective active control policy design for commensurate and incommensurate fractional order chaotic financial systems," Applied Mathematical Modelling. Simulation and Computation for Engineering and Environmental Systems, vol. 39, no. 2, pp. 500-514, 2015.

[20] C. Xie, Y. Xu, and D. Tong, "Chaos synchronization of financial chaotic system with external perturbation," Discrete Dynamics in Nature and Society. An International Multidisciplinary Research and Review Journal, Article ID 731376, Art. ID 731376, 7 pages, 2015.

[21] K. Ding and X. Xu, "Mixed synchronization of chaotic financial systems by using linear feedback control," Discrete Dynamics in
Nature and Society. An International Multidisciplinary Research and Review Journal, Article ID 3269248, Art. ID 3269248, 7 pages, 2016.

[22] Z. Wang and X. Huang, "Synchronization of a chaotic fractional order economical system with active control," in Proceedings of the 2011 International Conference on Advanced in Control Engineering and Information Science, CEIS 2011, pp. 516-520, chn, August 2011.

[23] C. Wang, H.-L. Zhang, and W.-H. Fan, “Generalized dislocated lag function projective synchronization of fractional order chaotic systems with fully uncertain parameters," Chaos, Solitons and Fractals, vol. 98, pp. 14-21, 2017.

[24] W. C. Chen, "Nonlinear dynamics and chaos in a fractionalorder financial system," Chaos, Solitons and Fractals, vol. 36, no. 5, pp. 1305-1314, 2008.

[25] M. Han and M.-L. Xu, "A hybrid prediction model of multivariate chaotic time series based on error correction," Acta Physica Sinica, vol. 62, no. 12, Article ID 120510, 2013.

[26] D. Xu and Z. Li, "Controlled projective synchronization in nonpartially-linear chaotic systems," International Journal of Bifurcation and Chaos in Applied Sciences and Engineering, vol. 12, no. 6, pp. 1395-1402, 2002.

[27] D. Matignon, "Stability result for fractional differential equations with applications to control processing," in Computational Engineering in Systems Applications, pp. 963-968, 1997.

[28] P. Zhou and W. Zhu, "Function projective synchronization for fractional-order chaotic systems," Nonlinear Analysis. Real World Applications. An International Multidisciplinary Journal, vol. 12, no. 2, pp. 811-816, 2011.

[29] D. S. Huang and H. Q. Li, Theory and method of the nonlinear economics publishing, House of Sichuan University, Chengdu, 1993. 


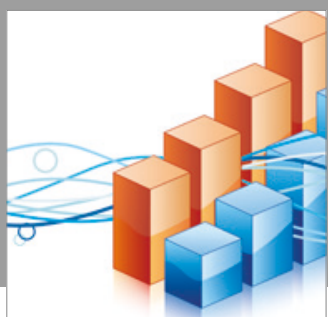

Advances in

Operations Research

vatersals

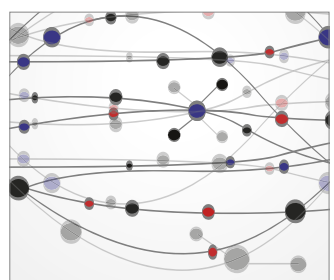

\section{The Scientific} World Journal
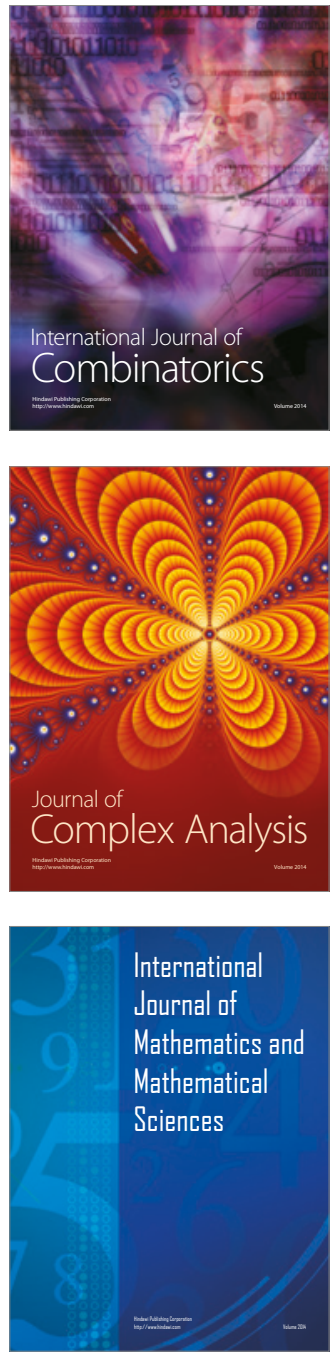
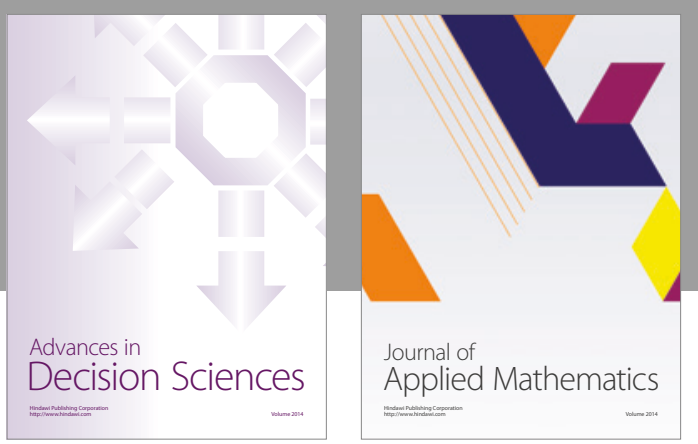

Algebra

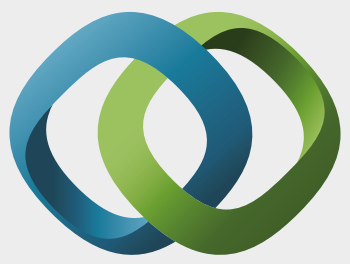

\section{Hindawi}

Submit your manuscripts at

https://www.hindawi.com
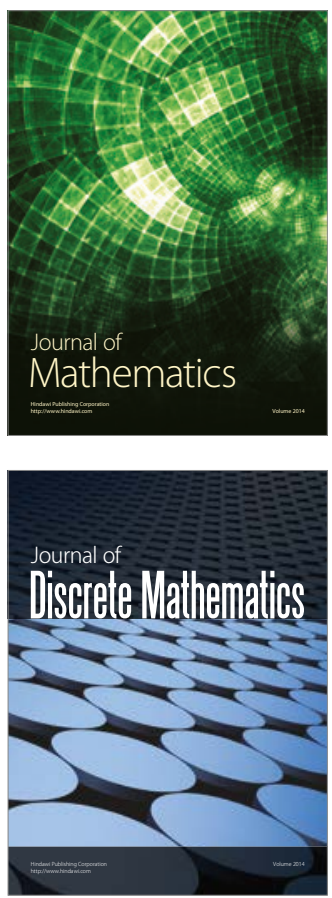

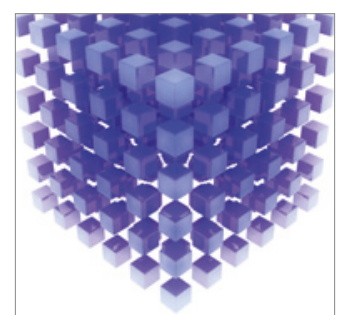

Mathematical Problems in Engineering
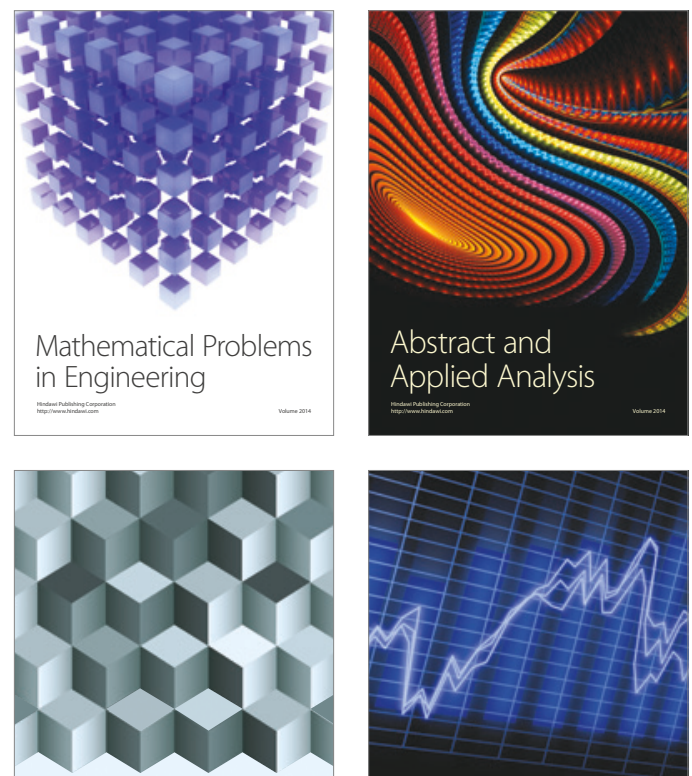

Journal of

Function Spaces

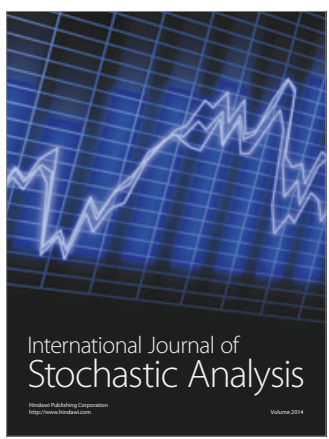

Probability and Statistics
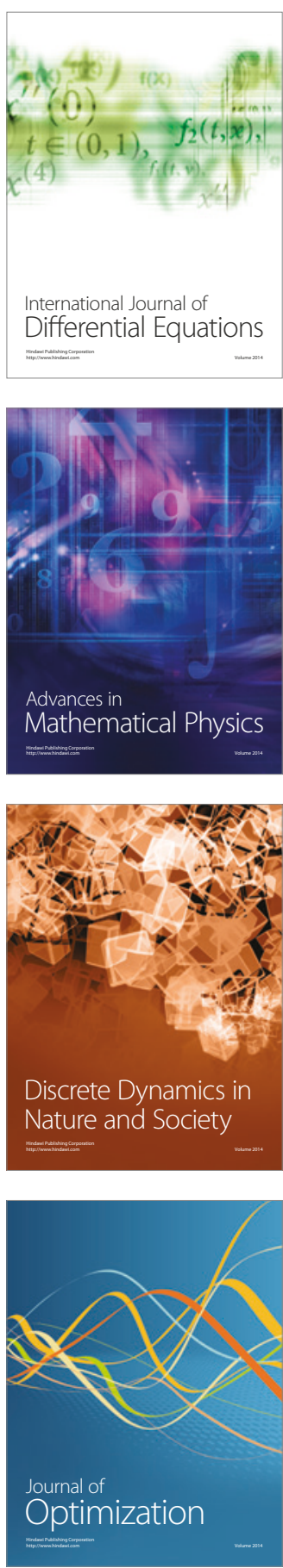